\title{
Printable Optical Filters for Visible Optical Communications
}

\author{
P. S. André, ${ }^{1,2}$ L. Nero ${ }^{1,3}$, Vânia T. Freitas ${ }^{2,4}$, M. S. Relvas ${ }^{2}$, R. A. S. Ferreira ${ }^{2,4}$ \\ ${ }^{1}$ Instituto de Telecomunicações, University of Aveiro, 3810-193 Aveiro, Portugal \\ ${ }^{2}$ Physic Department, University of Aveiro, 3810-193 Aveiro, Portugal \\ ${ }^{3}$ Electronics and Telecommunications Department, 3810-193 Aveiro, Portugal \\ ${ }^{4}$ CICECO, University of Aveiro, 3810-193 Aveiro, Portugal \\ Email: pandre@av.it.pt
}

Received 2013

\begin{abstract}
The design, production and characterization of tailored printable optical filters for visible optical communications are demonstrated. As result, the average color difference between the specified and the produced filters is 32.6, quantified in terms of CIELAB coordinates.
\end{abstract}

Keywords: Free-space Optical Communication; Wavelength Filtering Devices

\section{Introduction}

Visible Light Communication (VLC) is a latest technique in the field of wireless communications. This is a "Green" and energy-efficient emerging area, being developed by several researcher groups seeking high bandwidth, high security, and friendly communication networks using visible light instead of radio-frequency or microwave signals.

Previous studies have shown that it is possible to achieve a bandwidth of 500 Mbps [1]. The implementation of VLC systems using white light emission from commercial Light Emission Diodes (LED) was initial proposed by Komine and Nakagawa in 2004 [2]. The utilization of LED sources opens a window of opportunity for wavelength division multiplexing (WDM) [3]. However, the implementation of this solution requires cost effective optical filtering devices. Despite the commercial interest of the VLC technique, few works have been devoted to the subject [4].

In this work we propose the design of novel optical filters ink-jet printed in transparencies as a low cost solution for the large scale implementation of WDM VLC systems.

\section{Spectral Design}

The development of the commercial inkjet printers opens the possibility to create tailored high dynamic range optical filters. These printers are based on a subtractive color model, usually the CMYK model, which refers the 4 primary color used for printing, namely Cyan (C), Magenta (M), Yellow (Y) and blacK (K). For an accurate filter design it is essential a device color model that produces an estimate of the output characteristics for each combination of input parameters, typically performed by the printer color management system. Since we are interested in transmission optical filters printed in transparent substrates, the Murray-Davies model can be used, considering the spectral transmittance of the Neugebauer primaries and the Demichel equations [5]. However, the printing and the measurement of the Neugebauer primaries is impracticable for commercial inkjet systems. Other proposed approach is based on the principal component analyses but requiring a high computational calculus to obtained the final transfer function [6]. Therefore, we have implemented a distinct color model based on the Murray-Davies, but using the CMYK primaries.

In this approach, the filter average transmittance, $T(\lambda)$, is given by:

$$
\begin{aligned}
& T(\lambda)=T_{\text {substrate }}(\lambda) \prod_{j=1}^{4}\left\{\left(100-P_{j}\right) \max \left(T_{j}(\lambda)\right)+\right. \\
& \left.P_{j} \frac{T_{j}(\lambda)}{T_{\text {substrate }}(\lambda)}\right\}
\end{aligned}
$$

where $T_{j}(\lambda)$ refers to the spectral transmittance for each primary $(\mathrm{CMYK}), \mathrm{P}_{\mathrm{j}}$ is the primary intensity value (from 0 to 100$)$ and $T_{\text {substrate }}(\lambda)$ is the substrate spectral transmittance.

The 4 primary colors were printed in a BASF inkjet transparency with $200 \mu \mathrm{m}$ thickness, using an HP8100 inkjet printer. The spectral transmittance of each primary was measured using a dual-beam spectrometer Lambda 950, (Perkin-Elmer) with a $150 \mathrm{~mm}$ diameter Spectralon integrating sphere. These results were incorporated in 
equation 1 and used in an optimization routine, in order to estimate the primaries intensity values that produce a specific transmittance spectra. The optimization routine was implanted in Matlab and was based in the Levenberg-Marquardt algorithm.

\section{Implementation and Experimental Results}

For the test of this procedure a specific scenery was considered [4], using three optical filters with distinct features, in particular: band pass centered at i) $450 \mathrm{~nm}$ with $30 \mathrm{~nm}$ bandwidth (Blue) and at ii) $516 \mathrm{~nm}$ with $80 \mathrm{~nm}$ bandwidth (Green) and iii) a high wavelength pass filter with a transition edge at $586 \mathrm{~nm}$ (Red). The minimization routine was applied to these spectral profiles, in order to print sample substrates using the above mentioned inkjet printer. The transmittance spectra of these samples were measured. Figure 1 shows the transmittance spectra for the 3 optical filters termed as Red, Green and Blue. The inset in Figure 1 illustrates the samples color.

In order to visualize the color difference between the specified color and the measured one a uniform chromaticity scale color space CIE (u',v') chromaticity diagram was built using D65 illuminant and a $2^{\circ}$ standard observer, Figure 2.

The color difference between the specified one and the obtained filter transfer function was further quantified using the CIE ( $\mathrm{L}^{*}, \mathrm{a}^{*}, \mathrm{~b}^{*}$ ) color space (CIELAB, for the D65 illuminant and the $2^{\circ}$ standard observer). The measured (subscript $\mathrm{m}$ ) and specified (subscript s) transmittance spectra were quantified in terms of the parameters $\mathrm{L}, \mathrm{a}^{*}$ and $\mathrm{b}^{*}$ and the color difference obtained from:

$$
\Delta \mathrm{E}=\sqrt{\left(L_{m}-L_{s}\right)^{2}+\left(a_{m}-a_{s}\right)^{2}+\left(b_{m}-b_{s}\right)^{2}}
$$

The obtained color difference was 32, 31 and 35 for the Red, Blue and Green filters, respectively, resulting in

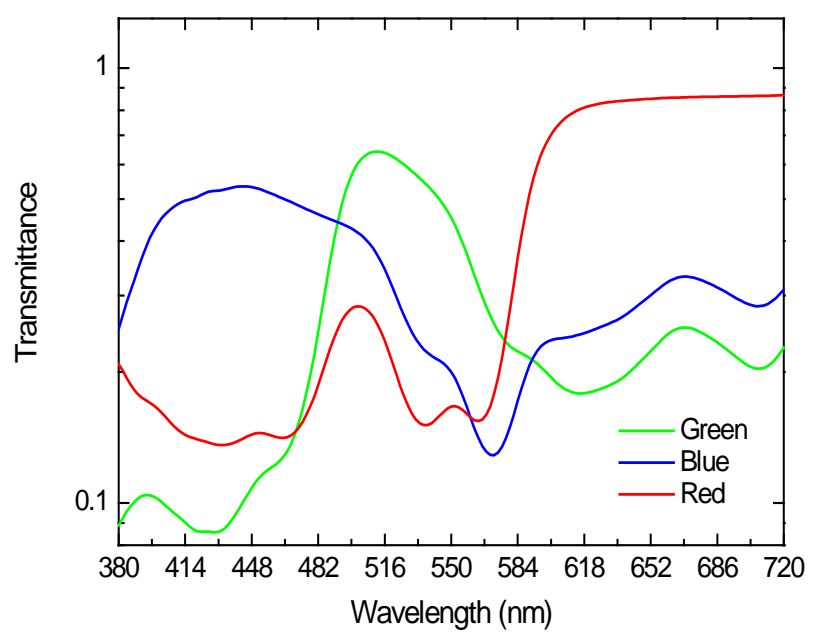

Figure 1. Transmittance spectra for the 3 optical filters designated as Red (R), Green (G) and Blue (B). The insets show the color appearance for the $R, G$ and $B$ filters.

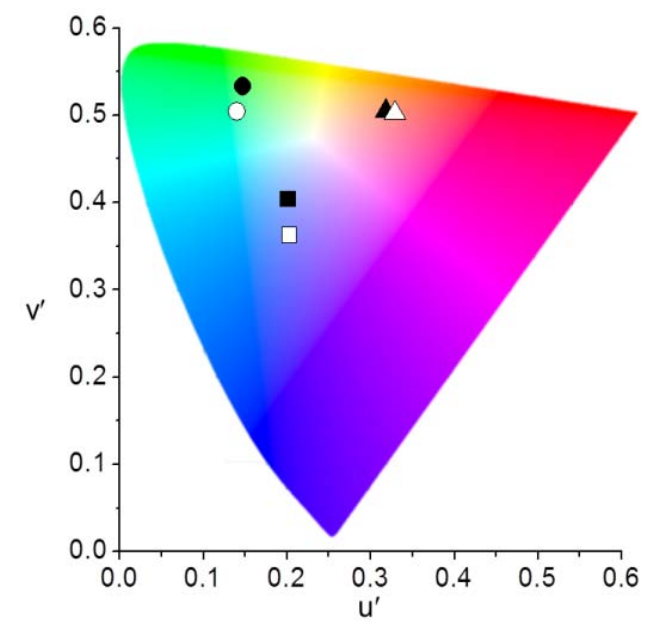

Figure 2. CIE (u',v') chromaticity diagram color for the specified (open symbols) and measured (solid symbols) optical filters transfer functions.

an average value of 32.6, which corresponds to a deviation of $6.8 \%$. This color difference between the measured and specified filters can by relate to the limited color Garmout of the inkjet printers. Nevertheless, the small value for the color mismatch $(6.8 \%)$ demonstrates that it is possible to attain a tailored spectral response suitable for the implementation of VLC WDM systems.

\section{Conclusions}

We have demonstrated the production feasibility of tailored printer optical filters. The filters transfer function was optimized using a color model based on the MurrayDavies, using the CMYK primaries. The average color difference between the specified and produced filters is 32.6, quantified in terms of CIELAB coordinates, which is a promising indicator about the potential of this low cost solution for visible free space optical communications.

\section{Acknowledgements}

The financial support of "Scientific and technological collaboration Portugal/China 2010/2012. Proc.0 441.00” is gratefully acknowledged.

\section{REFERENCES}

[1] W.-Y. Lin, C.-Y. Chen, H.-H. Lu, C.-H. Chang, Y.-P. Lin, H.-C. Lin and H.-W. Wu, "10m/500Mbps WDM Visible Light Communication Systems” Optics Express, Vol. 20, 2012, pp. 9919-9924.

[2] T. Komine and M. Nakagawa, "Fundamental Analysis for Visible Light Communication System Using LED Lights," IEEE Transactions on Consumer Electronics, Vol. 50, 2004, pp. 100-107. doi:10.1109/TCE.2004.1277847 
[3] K.Y. Cui, G. Chen, Q.F. He and Z.Y. Xu, "Indoor Optical Wireless Communication by Ultraviolet and Visible Light,"Proceedings of SPIE 7091, 2009, p. 709106.

[4] M. S. Uddin, J. S. Cha, J. Y. Kim and Y. M. Jang, "Mitigation Technique for Receiver Performance Variation of Multi-Color Channels in Visible Light Communication”, Vol. 11, 2011, pp. 6131-6144. doi:10.3390/s110606131

[5] I. Amidror and R. D. Hersch, "Neugebauer and Demichel: Dependence and Independence in N-Screen Superposi- tions for Colour Printing,” Color Research \& Application Vol. 25, 2000, pp. 267-277.

doi:10.1002/1520-6378(200008)25:4<267::AID-COL7 $>3$ .0.CO;2-M

[6] J. McElvaina, J. Millera and E. Jin, "Spectral Printer Modeling for Transparency Media: Toward High Dynamic Range Scene Reproduction”, Proceedings of SPIE, Vol. 7241, 2009, pp. 72410U-1.

doi:10.1117/12.806123 